Artigos

\title{
Avaliação de Parâmetros Físicos para Direção Veicular: Força, Audição e Visão
}

\author{
Assessment of Physical Parameters for Driving: \\ Strength, Hearing, and Vision
}

\author{
Yuri Franco Trunckle 1 , Cristina Akemi Okamoto ${ }^{1}$, Caroline Machado \\ Daitx ${ }^{1}$, Diego Toniolo do Prado ${ }^{1}$ Juliana Takitane ${ }^{1}$, \\ Daniel Romero Muñoz ${ }^{1}$
}

DOI: http://dx.doi.org/10.11606/issn.2317-2770.v25i2p63-72

\begin{abstract}
Trunckle YF, Okamoto CA, Daitx CM, Prado DT, Takitane J, Muñoz DR. Avaliação de Parâmetros Físicos para Direção Veicular: Força, Audição e Visão. Saúde, Ética \& Justiça. 2020;25(2):63-72.

RESUMO: De acordo com o Código de Trânsito Brasileiro (CTB), todo candidato à Carteira Nacional de Habilitação $(\mathrm{CNH})$ deve submeter-se ao exame de aptidão física e mental. Essa avaliação, a ser realizada por médicos especialistas em Medicina de Tráfego, é constituída por anamnese, exame físico geral, exames específicos e exames complementares, a critério médico. A avaliação específica envolve diversos aparelhos e sistemas, sendo composta pela avaliação oftalmológica, otorrinolaringológica, cardiorrespiratória, neurológica, do aparelho locomotor e avaliação dos distúrbios do sono. A partir do resultado do exame, o médico perito examinador de trânsito pode considerar o candidato como apto, apto com restrições, inapto temporário e inapto. Objetivos: avaliar os parâmetros de saúde (força da mão, visão e audição) em funcionários e alunos da Faculdade de Medicina da Universidade de São Paulo (FMUSP) com base na resolução 425 do Conselho Nacional de Trânsito (CONTRAN), que determina as normas para obtenção da CNH. Método: foi aplicado questionário padronizado, seguido por exame clínico direcionado, utilizando o equipamento Raizamed ${ }^{\circledR}$. Resultados: dos 70 participantes, 47 encontravam-se aptos para a direção veicular. Dos 23 que apresentavam alguma alteração ao exame, que os tornou temporariamente ou permanentemente inaptos, 2 tiveram problemas ao teste de campo visual; 15 , por força manual; 2, por tempo de reação ao ofuscamento; 2 , por combinação de campo visual com força manual; 1 , por alterações de acuidade visual e auditiva; e 1, por alteração de força manual, campo visual, acuidade visual e tempo de reação ao ofuscamento. Conclusão: os condutores em exame de direção veicular devem ser avaliados de forma cuidadosa e individual para que cada caso tenha suas peculiaridades observadas a contento.
\end{abstract}

DESCRITORES: Condução de Veículo; Força da Mão; Audição; Zumbido; Visão Ocular; Visão de Cores.

\footnotetext{
1. Universidade de São Paulo, Faculdade de Medicina, Departamento de Medicina Legal, Ética Médica e Medicina Social e do Trabalho

Endereço para correspondência: Yuri Franco Trunckle. E-mail: yuriftrunckle@gmail.com
} 


\section{INTRODUÇÃO}

$\mathrm{S}$ egundo o Código de Trânsito Brasileiro (CTB), todo candidato à habilitação deve submeter-se ao exame de aptidão física $\mathrm{e}$ mental (Art. 147), o qual é renovável a cada cinco anos ou a cada três anos, para condutores com mais de 65 anos de idade.

O exame de aptidão física e mental é realizado por médicos de tráfego e sua realização obedece ao disposto na Resolução n ${ }^{\circ} 425$ de 2012 do Conselho Nacional de Trânsito (CONTRAN). Essa resolução determina quais os procedimentos médicos exigidos para a obtenção da Carteira Nacional de Habilitação (CNH): anamnese, exame físico geral, exames específicos e exames complementares a critério médico. A avaliação específica envolve avaliação oftalmológica, otorrinolaringológica, cardiorrespiratória, neurológica, avaliação do aparelho locomotor e dos distúrbios do sono.

A avaliação mínima dos diversos aparelhos a ser realizada envolve:

na avaliação oftalmológica, o teste de acuidade visual e do campo visual, o teste de visão cromática e o teste de limiar de visão noturna e reação ao ofuscamento;

na avaliação otorrinolaringológica, a prova da voz coloquial;

na avaliação cardiorrespiratória, a verificação da pressão arterial e auscultas cardíaca e pulmonar;

na avaliação neurológica, a mobilidade ativa, passiva e reflexa, a coordenação motora, a força muscular, a sensibilidade profunda, a fala e as percepções; e

avaliação de distúrbios do sono (somente para candidatos às categorias $\mathrm{C}, \mathrm{D}$ e $\mathrm{E}$ ).

A partir do resultado do exame, o médico perito examinador de trânsito pode considerar o candidato como apto, apto com restrições, inapto temporário e inapto. Esse resultado leva em conta a presença de possíveis alterações à anamnese ou nos exames físicos e complementares que possam prejudicar a habilidade de conduzir um veículo com segurança, tanto para o condutor e os passageiros do veículo como para os demais usuários das vias.

$\mathrm{O}$ médico perito examinador deve ter rigor ao realizar o exame de aptidão física e mental, pois deve levar em conta, além dos interesses do examinado, o bem-estar da comunidade que ficará sujeita à direção do candidato. Ele deve procurar pela presença de alterações físicas que tenham impacto na capacidade de dirigir um veículo automotor, assim como alterações mentais ou emocionais, uma vez que o ato de dirigir é complexo, e mesmo tirar a atenção da via por 1,6-2,0 segundos já aumenta o risco de acidentes ${ }^{1,2,3}$.
Portanto, a importância do presente trabalho se dá pela falta de estudos que avaliem a frequência de alterações ao exame de aptidão para condutores de veículos automotores habilitados, além da ausência de dados sobre a correta aplicação do disposto na Resolução $n^{\circ} 425$.

\section{OBJETIVOS}

Objetivo Geral: avaliar os parâmetros de saúde (força da mão, visão e audição) em funcionários e alunos da Faculdade de Medicina da Universidade de São Paulo (FMUSP) com base na resolução $\mathrm{n}^{\circ} 425$ do CONTRAN, que determina as normas para obtenção da $\mathrm{CNH}$.

Objetivo Secundário: traçar $\mathrm{o}$ perfil socioeconômico e ocupacional dos participantes do estudo.

\section{MÉTODOS}

Foi realizado estudo descritivo, epidemiológico do tipo transversal, com informações coletadas de pessoas dentro do quadro de funcionários e de alunos de pósgraduação da Faculdade de Medicina da Universidade de São Paulo (FMUSP) no período de 30/11/2018 a 28/02/2019.

Os critérios de inclusão foram: indivíduos portadores de Carteira Nacional de Habilitação que concordaram em participar do estudo e assinaram o Termo de Consentimento Livre e Esclarecido. Foram excluídos os indivíduos não portadores de Carteira Nacional de Habilitação.

Para a obtenção dos dados, foi aplicado questionário padronizado, seguido por exame clínico direcionado, utilizando o equipamento Raizamed ${ }^{\circledR}$.

O questionário incluía dados como idade, sexo, grau de escolaridade, profissão, categoria de $\mathrm{CNH}$, data de validade da $\mathrm{CNH}$, presença de diabetes, hipertensão arterial sistêmica ou outras comorbidades e uso de medicamentos, além de queixa de zumbido.

$\mathrm{O}$ equipamento Raizamed $^{\circledR}$ faz avaliação de força manual em quilograma-força, acuidade auditiva em decibéis, acuidade visual, alinhamento central, visão cromática das cores semafóricas e campo visual. Ao fim do exame clínico, era questionado se os participantes se recordavam de ter realizado o exame completo durante os exames de aptidão física e mental para obtenção e renovação da $\mathrm{CNH}$.

A duração média desse procedimento foi de cerca de 15 minutos. Foi garantido que as respostas e os resultados do exame não seriam identificados ou associados a quem respondesse e não seriam enviados a nenhuma autoridade policial ou instituição de fiscalização. Além disso, o participante teve a liberdade de, se desejasse, suspender a qualquer momento a sua 
participação, retirando seu consentimento sem qualquer prejuízo.

Antes do início da aplicação do questionário e do exame clínico, foi apresentado o termo de consentimento livre e esclarecido, elaborado de acordo com as determinações da Resolução n ${ }^{\circ}$. 466/2012 do Conselho Nacional de Saúde, para que o participante lesse e, se concordasse com o termo, o preenchesse com o nome completo e a cidade.

Não houve conflitos de interesse.

\section{RESULTADOS E DISCUSSÃO}

\section{Dados sociodemográficos dos participantes}

Foram obtidos dados de 70 condutores entre funcionários e alunos da Faculdade de Medicina da Universidade de São Paulo. Destes, 32 (45,71\%) eram do sexo feminino e $38(54,29 \%)$, do sexo masculino. A idade variou entre 23 e 69 anos. A média de idade foi de 43,78, e a mediana foi de 45 . Os dados de distribuição da idade são exibidos no Gráfico 1 .

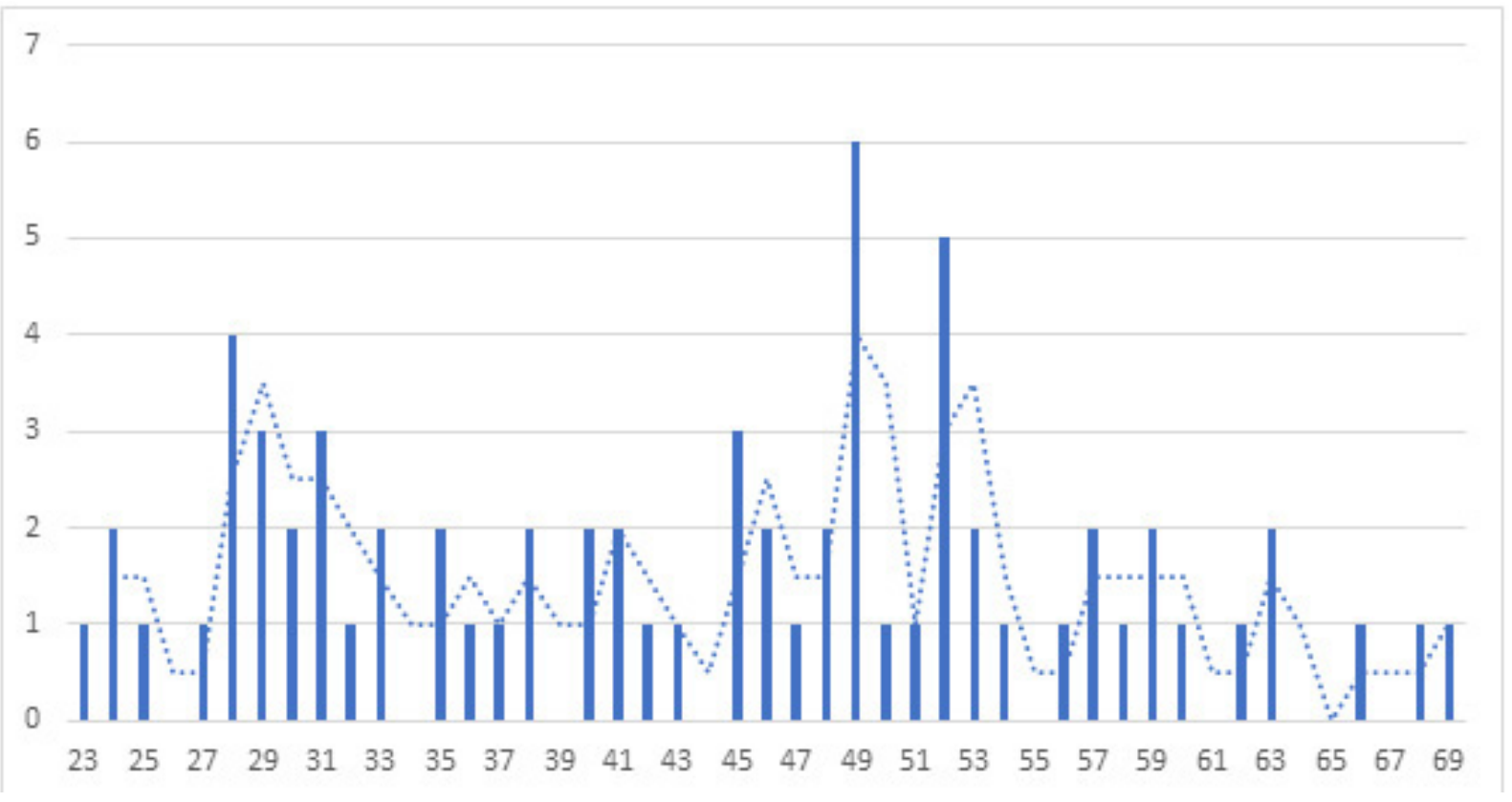

GRÁFICO 1 - Distribuição por idade

A Tabela 1 mostra o nível de escolaridade dos participantes. Observa-se que grande parte $(37,14 \%)$ tinha pós-graduação completa, o que pode ser atribuído ao fato de que os dados foram coletados na maioria entre os pesquisadores dos laboratórios e os alunos dos cursos de especialização da Faculdade de Medicina. Em 64 dos participantes foi constatado pelo menos educação de nível médio, o que corresponde a 91,42\%.

TABELA 1 - Distribuição dos participantes por escolaridade

\begin{tabular}{l|l|l}
\hline Escolaridade & Número absoluto & Porcentagem \\
\hline Ensino fundamental incompleto & 2 & $2,86 \%$ \\
\hline Ensino fundamental completo & 2 & $2,86 \%$ \\
\hline Ensino médio incompleto & 2 & $2,86 \%$ \\
\hline Ensino médio completo & 18 & $25,71 \%$ \\
\hline Ensino superior incompleto & 5 & $7,14 \%$ \\
\hline Ensino superior completo & 7 & $10,00 \%$ \\
\hline Pós-graduação incompleta & 8 & $11,43 \%$ \\
\hline Pós-graduação completa & 26 & $37,14 \%$ \\
\hline
\end{tabular}


Conforme mostram os dados da Tabela 2, a profissão mais frequente entre os participantes foi de médico. Isso se deu, como já foi mencionado, pelo fato de grande parte dos participantes serem alunos dos cursos de especialização da Faculdade de Medicina. Outras profissões incluem os pesquisadores dos laboratórios da Faculdade e os funcionários da manutenção ou vigilância.

TABELA 2 - Distribuiç̧ão por profissão

\begin{tabular}{|c|c|c|}
\hline Profissão & $\begin{array}{l}\text { Número } \\
\text { absoluto }\end{array}$ & Porcentagem \\
\hline Médico & 13 & $18,57 \%$ \\
\hline Biólogo & 6 & $8,57 \%$ \\
\hline Secretário & 6 & $8,57 \%$ \\
\hline Eletricista & 5 & $7,14 \%$ \\
\hline Técnico de laboratório & 4 & $5,71 \%$ \\
\hline Assistente de gerência & 3 & $4,29 \%$ \\
\hline Biomédico & 2 & $2,86 \%$ \\
\hline Encarregado de setor & 2 & $2,86 \%$ \\
\hline Estudante & 2 & $2,86 \%$ \\
\hline Pedreiro & 2 & $2,86 \%$ \\
\hline Técnico de manutenção & 2 & $2,86 \%$ \\
\hline Ajudante de obras & 1 & $1,43 \%$ \\
\hline Analista de sistemas & 1 & $1,43 \%$ \\
\hline Auxiliar acadêmico & 1 & $1,43 \%$ \\
\hline Auxiliar de laboratório & 1 & $1,43 \%$ \\
\hline Bombeiro civil & 1 & $1,43 \%$ \\
\hline Farmacêutico & 1 & $1,43 \%$ \\
\hline Mecânico de ar condicionado & 1 & $1,43 \%$ \\
\hline Operador & 1 & $1,43 \%$ \\
\hline Operador de áudio e vídeo & 1 & $1,43 \%$ \\
\hline Operador de caldeira & 1 & $1,43 \%$ \\
\hline Pintor & 1 & $1,43 \%$ \\
\hline Professora & 1 & $1,43 \%$ \\
\hline Psicólogo & 1 & $1,43 \%$ \\
\hline Técnico administrativo & 1 & $1,43 \%$ \\
\hline Técnico de áudio e vídeo & 1 & $1,43 \%$ \\
\hline Outros & 8 & $11,43 \%$ \\
\hline
\end{tabular}

\section{Dados da Carteira Nacional de Habilitação (CNH) dos participantes}

Foram obtidos os dados referentes a data de validade, categoria e restrições das Carteiras Nacionais de Habilitação $(\mathrm{CNH})$ dos participantes. Os dados correspondentes são mostrados na Tabela 3. Somente um dos entrevistados possuía $\mathrm{CNH}$ com vencimento em 2003.

A maior parte dos condutores entrevistados (77,14\%) possuía $\mathrm{CNH}$ com categoria $\mathrm{B}$, correspondente aos automóveis comuns. Em seguida, vêm os com 
condutores com permissão para dirigir na categoria $\mathrm{AB}(11,43 \%)$, que abrange além dos automóveis, as motocicletas. A minoria tinha habilitação para os veículos maiores, como caminhões e ônibus

A maioria dos participantes não apresentava restrições para direção veicular $(71,43 \%)$. A restrição mais comum entre os demais foi a " $\mathrm{A}$ " (obrigatório uso de lentes corretivas), correspondendo a $25,71 \%$ da amostra. Uma pessoa possuía restrição "B" (obrigatório uso de prótese auditiva); uma possuía restrição " $G$ " (obrigatório o uso de veículo com embreagem manual ou com automação de embreagem ou com transmissão automática); duas possuíam restrição "D" (obrigatório o uso de veículo com transmissão automática); e uma possuía restrição "F" (obrigatório o uso de veículo com direção hidráulica).

TABELA 3 - Dados da CNH dos participantes

\begin{tabular}{|c|c|c|}
\hline Data de validade & Número absoluto & Porcentagem \\
\hline 2003 & 1 & $1,43 \%$ \\
\hline 2019 & 5 & $7,14 \%$ \\
\hline 2020 & 16 & $22,86 \%$ \\
\hline 2021 & 14 & $20,00 \%$ \\
\hline 2022 & 15 & $21,43 \%$ \\
\hline 2023 & 13 & $18,57 \%$ \\
\hline 2024 & 1 & $1,43 \%$ \\
\hline 2025 & 1 & $1,43 \%$ \\
\hline 2026 & 0 & $0,00 \%$ \\
\hline 2027 & 1 & $1,43 \%$ \\
\hline Não se recorda & 3 & $4,29 \%$ \\
\hline \multicolumn{3}{|l|}{ Categoria } \\
\hline $\mathrm{AB}$ & 8 & $11,43 \%$ \\
\hline $\mathrm{B}$ & 54 & $77,14 \%$ \\
\hline $\mathrm{C}$ & 4 & $5,71 \%$ \\
\hline $\mathrm{D}$ & 3 & $4,29 \%$ \\
\hline $\mathrm{E}$ & 1 & $1,43 \%$ \\
\hline \multicolumn{3}{|l|}{ Restrição } \\
\hline Não tem restrição & 50 & $71,43 \%$ \\
\hline A & 18 & $25,71 \%$ \\
\hline $\mathrm{B}$ & 1 & $1,43 \%$ \\
\hline $\mathrm{G}$ & 1 & $1,43 \%$ \\
\hline $\mathrm{D}$ & 2 & $2,86 \%$ \\
\hline $\mathrm{F}$ & 1 & $1,43 \%$ \\
\hline
\end{tabular}

\section{Dados de doenças prévias e uso de medicações nos participantes}

$\mathrm{Na}$ entrevista realizada com os participantes, foi questionado o histórico pessoal de comorbidades.
Questionou-se especificamente sobre diabetes mellitus e hipertensão arterial sistêmica, além de outras doenças e uso de medicamentos.

Quanto às comorbidades, $7(10,00 \%)$ participantes possuíam diabetes mellitus e $12(17,14 \%)$, hipertensão 
arterial sistêmica. Para Schmidt et al., em seu estudo, a prevalência de diabetes autorreferida na população brasileira é de 5 a $6 \%$, e de hipertensão é entre 21,6 e $24,4 \%{ }^{4}$. Tais achados entram em concordância com a alta prevalência destas doenças na população brasileira e trazem implicações na direção veicular, na forma de complicações agudas ou crônicas. Essas comorbidades também aumentam consideravelmente com a idade, o que justifica um menor tempo entre as avaliações na população idosa bem como a necessidade de um exame apurado. Além disso, 10 (14,29\%) referiram outras comorbidades: dislipidemia $(n=2)$, Chagas e câncer, miocardite viral há 10 anos, hérnia discal, fibromialgia, asma, perda auditiva à esquerda, talassemia, trombose venosa profunda ( $\mathrm{n}=1$, para cada condição).

A maioria dos participantes $(n=46,65,71 \%)$ negou uso de medicamentos. Dentre os demais, os medicamentos relatados foram: antidepressivos (fluoxetina, venlafaxina, escitalopram), anti-hipertensivos (losartana, indapamida, candesartana, valsartana, hidroclorotiazida, atenolol, enalapril, verapamil), medicamentos utilizados em casos de insuficiência cardíaca (carvedilol, monocordil, hidralazina), asma (mometasona e salbutamol), dislipidemia (sinvastatina), anticoncepcionais, dentre outros (cálcio, vitamina D, tamoxifeno, omeprazol).

\section{Exame clínico (Raizamed ${ }^{\circledR}$ )}

Foi utilizado o equipamento da marca Raizamed ${ }^{\circledR}$ para a realização do exame clínico. Foram testados os parâmetros: força manual, acuidade auditiva, acuidade visual, estereopsia por meio do teste de alinhamento central, tempo de reação ao ofuscamento, campo visual e visão cromática.

Os resultados do teste de força manual são exibidos no Gráfico 2. A maioria dos participantes apresentou força de $20 \mathrm{kgf}$ em ambos os membros, em geral com resultados de força superiores no membro direito, provavelmente pelo predomínio de destros na população. Esse resultado atende, portanto, um requisito mínimo obrigatório de força para obtenção da $\mathrm{CNH}$. No teste do membro superior direito há um resultado a menos, porque um dos participantes não realizou o teste com a mão.

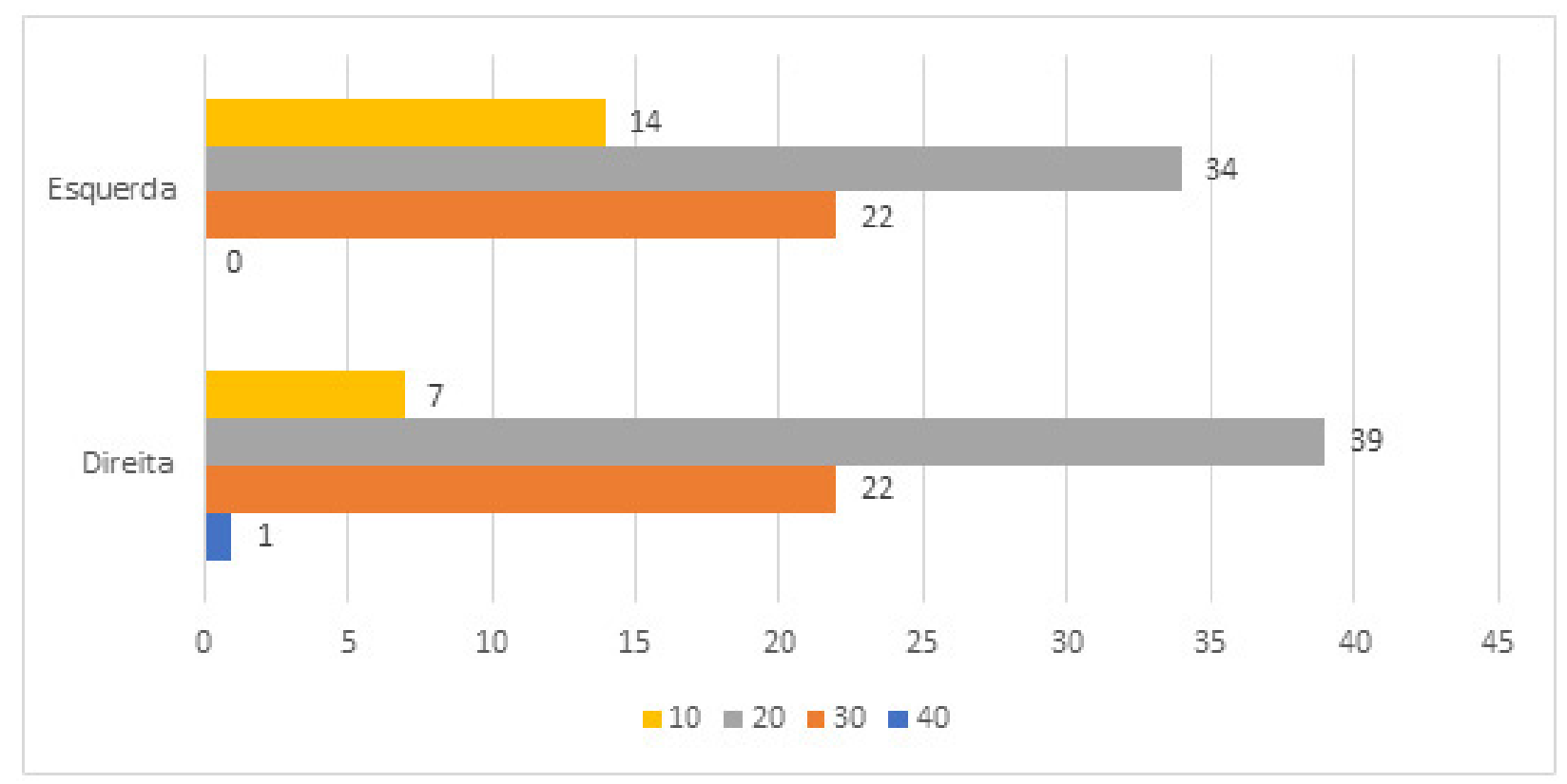

GRÁFICO 2 - Força manual

No teste de acuidade auditiva, inicialmente foi questionado se os participantes apresentavam queixa de zumbido: 64 negaram, 2 queixaram-se de zumbido à direita somente e 4 queixaram-se de zumbido bilateral. Foi então avaliada a acuidade auditiva pelo equipamento da Raizamed ${ }^{\circledR}$. A grande maioria $(n=68,97,14 \%)$ apresentou limiar auditivo inferior a $30 \mathrm{~dB}$, como observado no Gráfico 3.

Tem-se, portanto, uma conformidade com o limite estabelecido de acordo com o Anexo III da Resolução, que estabelece como perda máxima de $40 \mathrm{~dB}$ para condução de veículo em qualquer categoria.

Os resultados de acuidade visual são apresentados no Gráfico 4, sendo que $61,43 \%$ à esquerda e $70,00 \%$ à direita apresentaram acuidade visual de 20/20 tendo, portanto, a mínima necessária de acordo com a legislação.

Vale ressaltar que de acordo com o Anexo II da legislação em questão, para condutores de veículos de 
categoria ACC, A e B, é preciso "acuidade visual central igual ou superior a 20/40 (equivalente a 0,50) em cada um dos olhos ou igual ou superior a 20/30 (equivalente a 0,66) em um dos olhos, com pelo menos percepção luminosa (PL) no outro".

Por sua vez, nas categorias C, D e E consta: "Acuidade visual central igual ou superior a 20/30 (equivalente a 0,66) em cada um dos olhos ou igual ou superior a 20/30 (equivalente a 0,66) em um olho e igual ou superior a 20/40 (equivalente a 0,50) no outro, com visão binocular mínima de 20/25 (equivalente a 0,80)".

Dos que apresentaram diminuição da acuidade visual, $87,14 \%$ bilateralmente apresentavam no mínimo 20/30 e $95,71 \%$ bilateralmente, $20 / 40$.

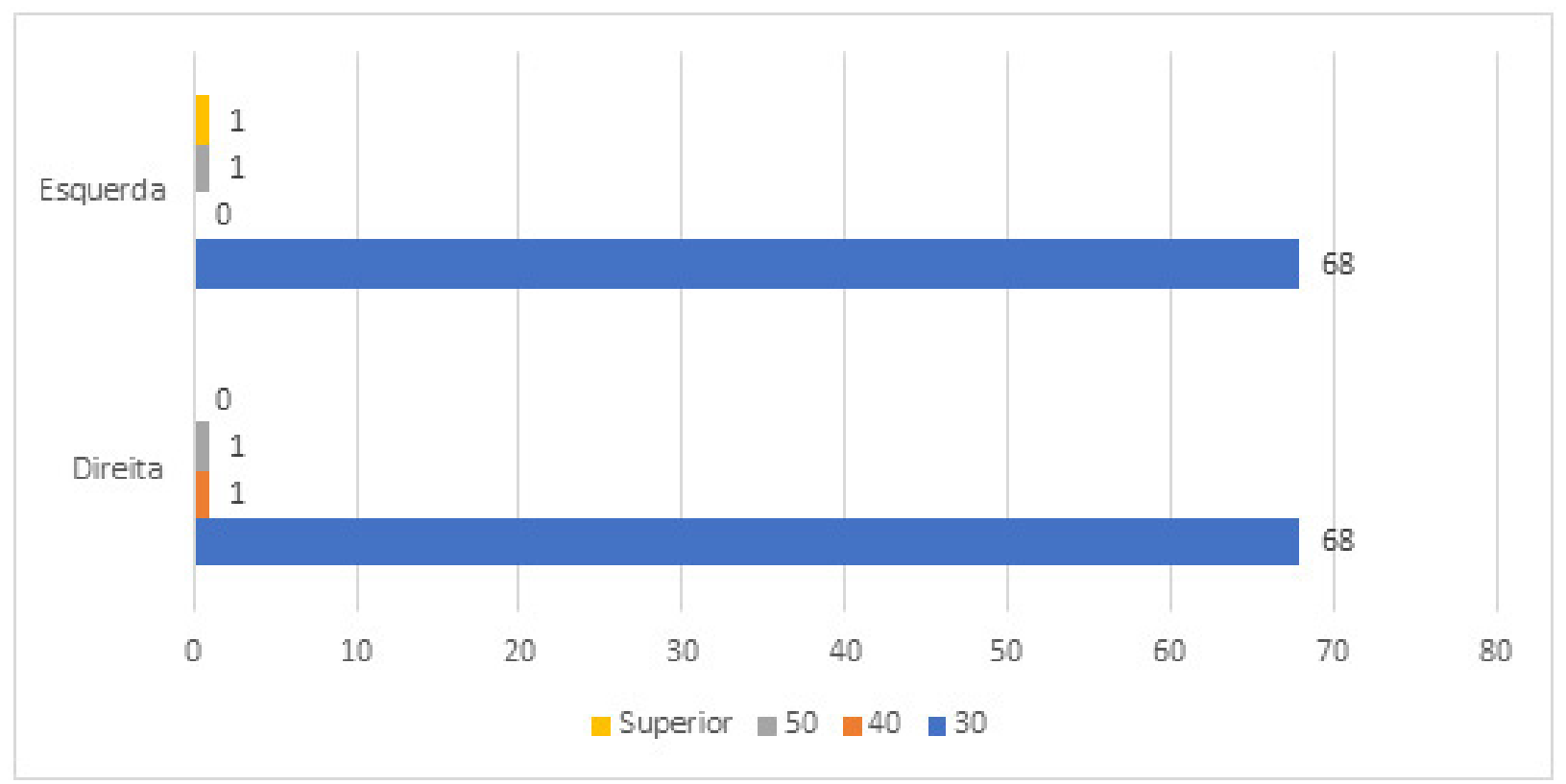

GRÁFICO 3 - Acuidade auditiva

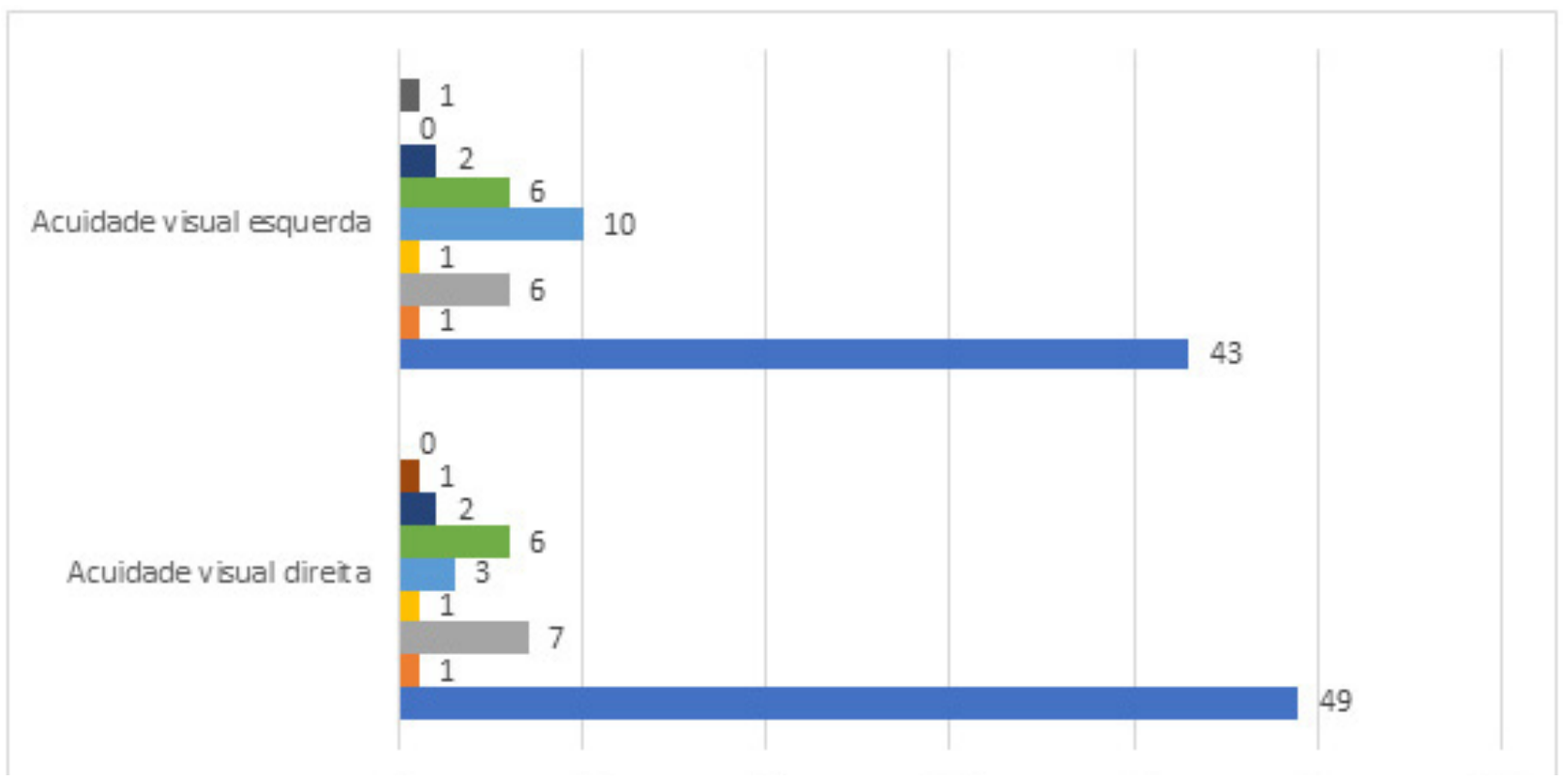

GRÁFICO 4 - Acuidade visual

A estereopsia foi avaliada por meio do alinhamento central, a qual, no entanto, não é contemplada pela resolução $n^{0} 425 / 2012$ do CONTRAN; portanto, o resultado desse teste não foi utilizado para avaliação de aptidão para a condução veicular realizada ao fim do estudo. Dezessete dos participantes $(24,28 \%$ ) não conseguiram fazer o alinhamento central, como exibido no Gráfico 5. 


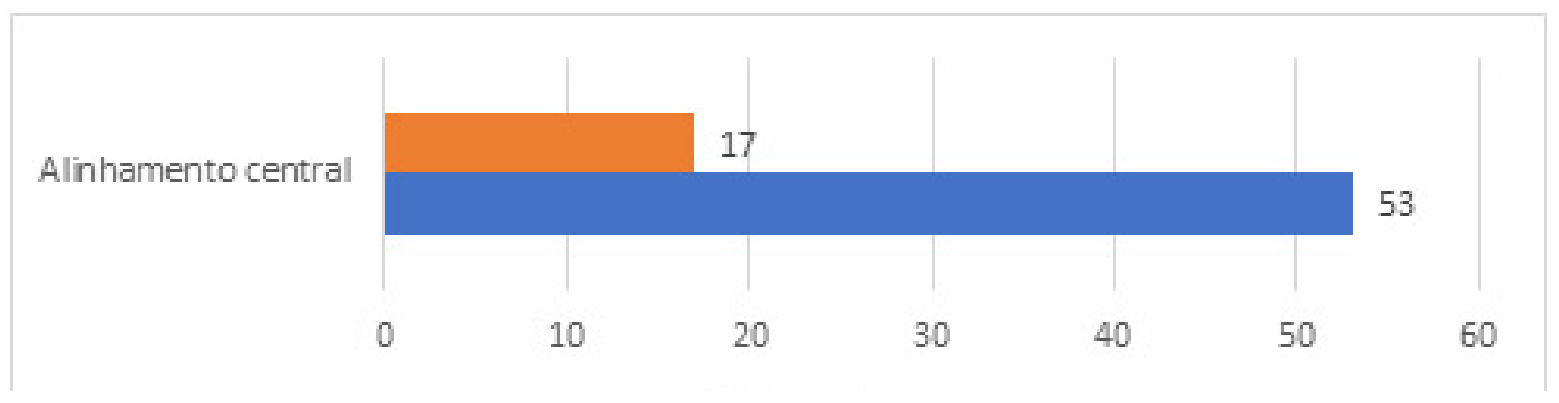

GRÁFICO 5 - Alinhamento central

Foi também realizado teste de reação ao ofuscamento. Como a resolução $n^{\circ} 425 / 2012$ do CONTRAN não define valores para a normalidade deste teste, foi uti- lizado valor de até três segundos. Como observado no Gráfico 6, somente 4 pessoas $(5,71 \%)$ apresentaram tempo de reação ao ofuscamento superior a três segundos.

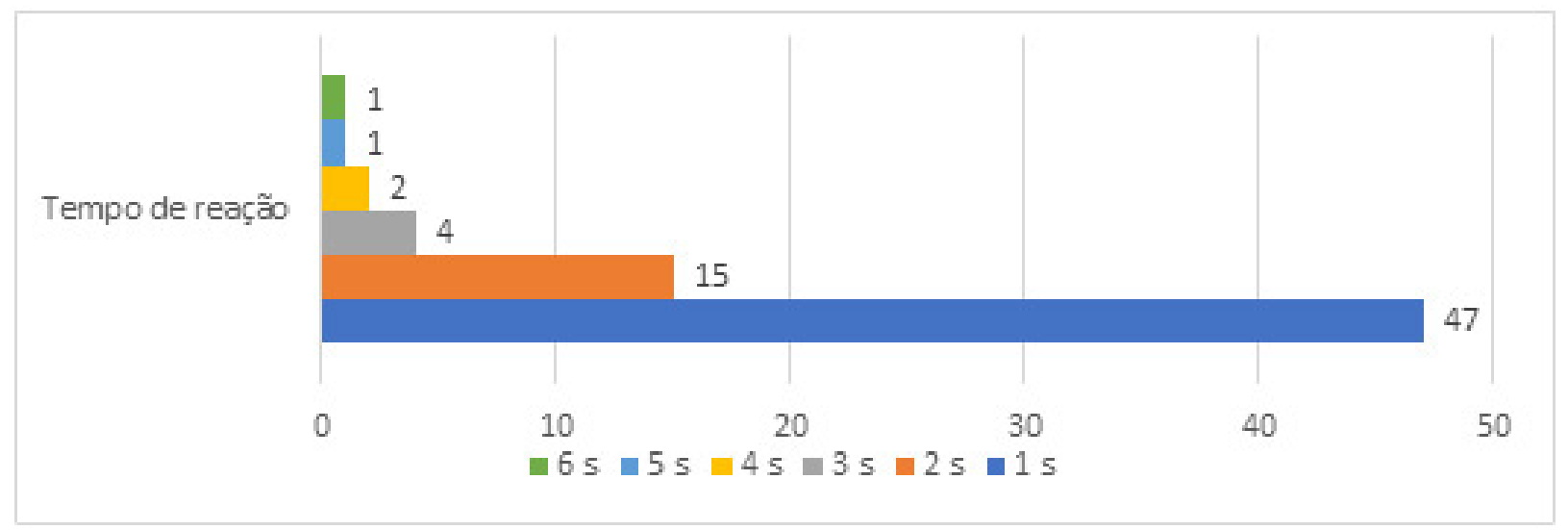

GRÁFICO 6 - Tempo de reação ao ofuscamento

O Gráfico 7 exibe os resultados da avaliação de campo visual. Somente 2 pessoas apresentaram campo menor que $60^{\circ}$ à direita, e 3 apresentaram à esquerda, tendo como parâmetro a resolução citada anteriormente, a qual define como mínimo necessário visão periférica igual ou superior a $60^{\circ} \mathrm{em}$ cada um dos olhos ou igual ou superior a $120^{\circ}$ em um olho.

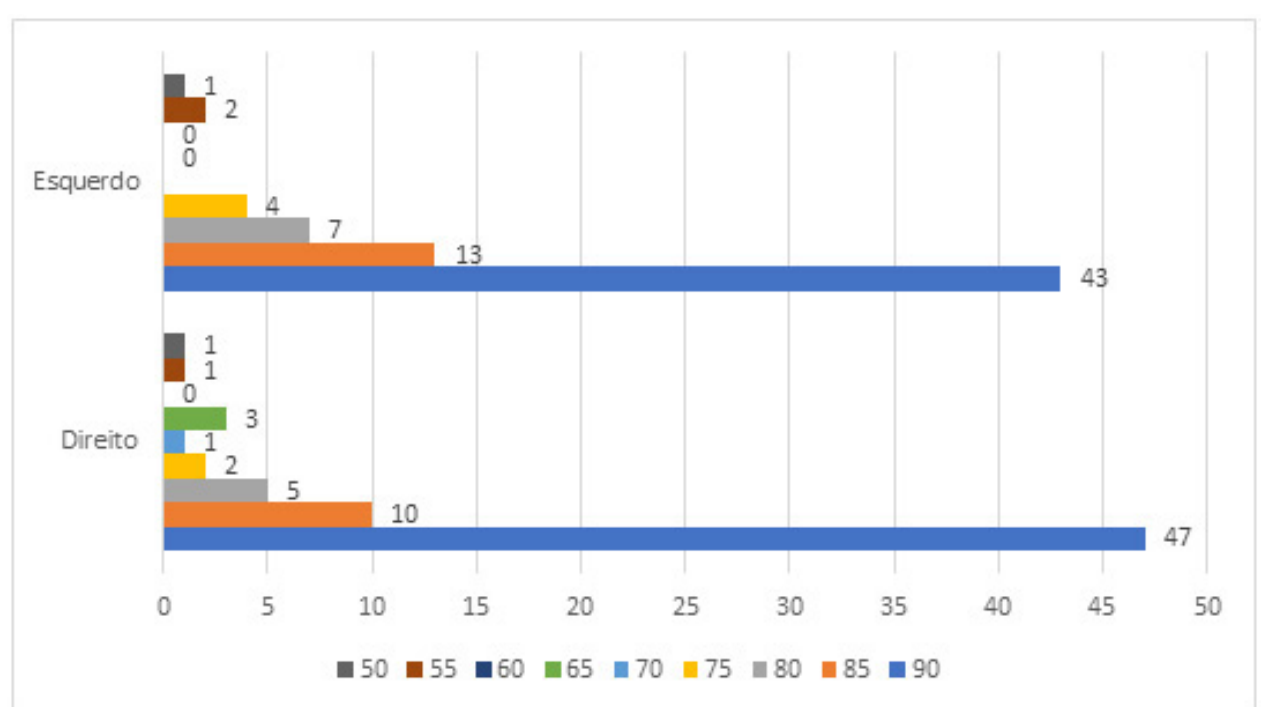

GRÁFICO 7 - Campo visual 
Foi realizado ainda teste de visão cromática, cujas alterações também não são impeditivas à condução veicular, sendo necessário somente reconhecimento de luzes semafóricas em posição padronizada. Neste teste, somente duas pessoas apresentaram alterações, ambas com dificuldade em identificar a cor amarela.

\section{Resultados dos exames quanto à aptidão para condução veicular}

Foram analisados os resultados obtidos pelo exame clínico e a categoria de $\mathrm{CNH}$ do participante, com base na resolução $n^{\circ}$ 425/2012 do CONTRAN.

Dos 70 participantes, 47 encontravam-se aptos para a direção veicular. Dos 23 que apresentaram alguma alteração ao exame que os tornou temporariamente ou permanentemente inaptos, 2 foram devido ao teste de campo visual; 15 , por força manual; 2 , por tempo de reação ao ofuscamento; 2 , por combinação de campo visual com força manual; 1, por alterações de acuidade visual e auditiva; e 1, por alteração de força manual, campo visual, acuidade visual e tempo de reação ao ofuscamento.

\section{Dados sobre os exames de aptidão física e mental realizados na habilitação ou renovação da $\mathrm{CNH}$ dos participantes}

Foi questionado após a realização do exame clínico se os participantes se recordavam de ter realizado o exame completo na ocasião em que solicitaram a primeira habilitação ou em que renovaram a $\mathrm{CNH}$. Somente quatro pessoas $(6,71 \%)$ relataram ter realizado todos os testes requeridos pela Resolução $n^{\circ} 425 / 2012$ do CONTRAN para habilitação de condutores, observando que dentre os testes realizados, não há obrigatoriedade do alinhamento central e da visão de cores, somente reconhecimento de luzes semafóricas em posição padronizada.

Entre os testes mais frequentemente realizados nos exames de aptidão física e mental está a acuidade visual, que foi realizada em $80 \%$ dos participantes. Os demais testes não foram aplicados em mais de 45 $(64,29 \%)$ dos participantes, sendo o menos aplicado o de campo visual $(\mathrm{n}=59,84,29 \%)$. Esses dados são apresentados na Tabela 4.

TABELA 4 - Testes não realizados durante os exames de aptidão para candidatos à habilitação ou renovação, conforme relatado pelos participantes

\begin{tabular}{l|c|c}
\hline Teste não realizado & Número & Porcentagem \\
\hline Força manual & 51 & $72,86 \%$ \\
\hline Acuidade auditiva & 50 & $71,43 \%$ \\
\hline Acuidade visual & 14 & $20,00 \%$ \\
\hline Alinhamento & 58 & $82,86 \%$ \\
\hline Ofuscamento & 45 & $64,29 \%$ \\
\hline Campo visual & 59 & $84,29 \%$ \\
\hline Visão cromática & 46 & $65,71 \%$ \\
\hline
\end{tabular}

Os condutores em exame de direção veicular devem ser avaliados de forma cuidadosa e individual para que cada caso tenha suas peculiaridades observadas a contento. No caso de condutores com comorbidades mais comuns, como diabetes mellitus ou hipertensão, em especial os condutores de veículos destinados a transporte de cargas e passageiros, devem passar por uma avaliação abrangente com o médico de tráfego.

Em nossa amostra, a minoria dos participantes conduziam veículos de passageiros ou cargas, sendo assim limitada à associação em nosso estudo de atividade profissional e comorbidades. Ainda que nessa amostra os partícipes não se utilizem de veículos para proverem seu sustento, a importância do uso de veículos de transporte na rotina dos brasileiros é indiscutível, tendo em vista a precariedade dos transportes coletivos em nosso país e seu impacto na mobilidade urbana.

\section{CONCLUSÃO}

Nossa amostra demonstra que uma parcela significativa dos condutores não apresentava os parâmetros de saúde necessários à condução, avaliados de acordo com a Resolução ${ }^{\circ} 425$ do CONTRAN. A maioria decorreu de alterações de força muscular manual isoladamente, o que coincide com o teste menos realizado nos exames de aptidão. Isso reforça a importância de o exame de direção ser realizado de maneira completa e criteriosa.

Foi também constatado que a maioria dos condutores do estudo estava na quinta década de vida (40-50 anos), é habilitada apenas para veículos de passeio (categoria B) e possui escolaridade acima do ensino médio. 
Trunckle YF, Okamoto CA, Daitx CM, Prado DT, Takitane J, Muñoz DR. Assessment of Physical Parameters for Driving: Strength, Hearing, and Vision. Saúde, Ética \& Justiça. 2020;25(2):63-72.

ABSTRACT: Introduction: According to the Brazilian Traffic Code (CTB), every person applying for the National Driver's License $(\mathrm{CNH})$ must undergo the physical and mental aptitude tests. This evaluation, carried out by a physician specialized in Traffic Medicine, consists of an anamnesis, general physical examination, specific examinations and complementary examinations at the physician's discretion. The specific evaluation involves several devices and systems; it includes the ophthalmological, otolaryngological, cardiorespiratory, and neurological, locomotor system and sleep disorder assessments. Based on the result of the tests, the medical expert can consider the applicant fit, fit with restrictions, temporarily unfit and unfit. Objectives: to evaluate health parameters (hand strength, vision and hearing) in employees and students of the University of São Paulo School of Medicine (FMUSP) based on Resolution 425 of the National Traffic Council (CONTRAN), which determines the rules for obtaining the CNH. Method: a standardized questionnaire was applied, followed by a directed clinical test, using the Raizamed ${ }^{\circledR}$ equipment. Results: of the 70 participants, 47 were able to drive. Of the 23 who presented alterations in the tests that made them temporarily or permanently unfit, 2 had problems with the visual field test; 15 with manual strength; 2 with glare reaction time; 2 with a combination of the visual field and manual strength tests; 1 with changes in visual and auditory acuity; and 1 with alterations in manual strength, visual field, visual acuity and reaction time to glare. Conclusion: drivers undergoing driving tests must be carefully and individually assessed so that each case has its peculiarities fully observed.

KEY WORDS: Automobile driving; Hand strength; Hearing; Tinnitus; Vision, ocular; Color vision.

\section{REFERÊNCIAS}

1.Horrey WJ, Wickens CD. In-vehicle glance duration: distributions, tails, and a model of crash risk. Transp Res Rec. 2007;2018(1):22-8. DOI: https://doi.org/10.3141/2018-04

2.Klauer SG, Dingus TA, Neale VL, Sudweeks JD, Ramsey DJ. The impact of driver inattention on near-crash/crash risk: an analysis using the 100-Car Naturalistic Study Data. Technical Report. Washington (DC): National Highway Traffic Safety Administration, Office of Human-Vehicle Performance Research; 2006 Apr. Report No.: DOT HS 810594.

3.Simons-Morton BG, Guo F, Klauer SG, Ehsani JP, Pradhan AK. Keep your eyes on the road: young driver crash risk increases according to duration of distraction. $\mathrm{J}$ Adolesc Health. 2014;54(5Suppl):S61-7. DOI: https://doi. org/10.1016/j.jadohealth.2013.11.021

4.Schmidt MI, Duncan BB, Hoffmann JF, de Moura L, Malta DC, de Carvalho RMSV. Prevalência de diabetes e hipertensão no Brasil baseada em inquérito de morbidade auto-referida, Brasil, 2006. Rev Saúde Pública [Internet]. 2009 [Acesso em 2019 mai. 10];43(Supl 2):74-82. Disponível em: http:// www.scielo.br/scielo.php?script $=$ sci_arttext\&pid $=$ S0034 89102009000900010\&lng=en. DOI: https://doi.org/10.1590/ S0034-89102009000900010 